\title{
Dietary fat intake not associated with risk of breast cancer: analysis from the UK Women's Cohort Study
}

\author{
X. L. Nai ${ }^{1}$, K. L. Edwards ${ }^{2}$, V. J. Burley ${ }^{3}$, D. C. Greenwood ${ }^{4}$ and J. E. Cade ${ }^{3}$ \\ ${ }^{1}$ The Human Nutrition Unit, University of Sheffield, Sheffield S10 2RX, UK, ${ }^{2}$ Cancer Epidemiology Group, University \\ of Leeds, Leeds LS2 9JT, UK, ${ }^{3}$ Nutritional Epidemiology Group, University of Leeds, 71-75 Clarendon Road, \\ Leeds LS2 9PL, UK and ${ }^{4}$ Biostatistics Unit, University of Leeds, 24 Hyde Terrace, Leeds LS2 9LN, UK
}

Breast cancer is the most common diagnosed malignancy ${ }^{(1)}$ among women and it afflicts approximately 40000 women in the UK each year $^{(2)}$. The role of diet in affecting breast cancer risk is of great interest as a potentially modifiable risk factor. Findings from studies investigating the role of dietary fat intake in the aetiology of breast cancer development remain equivocal. While ecological ${ }^{(3)}$ and animal $^{(4)}$ studies suggested a direct positive fat-breast cancer association, evidences from observational epidemiological studies are inconsistent; most case-control studies have demonstrated a modest positive association ${ }^{(5)}$, whereas cohort studies provided little ${ }^{(6)}$ or no $^{(7)}$ support for fat-breast cancer association.

Thus, the aim of present study was to investigate the association between intakes of dietary fat and fat subtypes and breast cancer risk in a large cohort of middle-aged women in the UK.

In this UK Women's Cohort Study (UKWCS), a total of 35372 women aged 35-69 years were recruited between 1995 and 1998. Of these, 15991 were pre-menopausal women and 17781 were postmenopausal women. Sub-sample analysis was performed for family history of breast cancer ( $n$ 18 297). During the mean follow-up of 9 years, 876 women developed invasive breast carcinoma; with 360 and 501 were pre- and post-menopausal women; as well as 47 and 412 were women with and without family history of breast cancer, respectively. Dietary fat and fat subtypes intakes were assessed using a 217-item Food Frequency Questionnaire. Cox proportional hazards models, adjusted for known confounders, were used to estimate hazard ratios (HR) for breast cancer. Association was estimated for the entire cohort, for pre- and post-menopausal women and for women with and without family history of breast cancer separately.

No significant association of breast cancer with total, SFA, MUFA or PUFA was found in the entire population, although trends were for a positive association of risk with total, SFA and MUFA and an inverse association with PUFA. In pre-menopausal women, nonsignificant positive correlation was observed for total fat and all fat subtypes, whereas in post-menopausal women, this positive relationship was only observed for saturated fat. In women with family history of breast cancer, MUFA and PUFA were insignificantly positively associated with breast cancer, but insignificantly negatively associated with breast cancer in women without family history.

\begin{tabular}{|c|c|c|c|c|c|c|}
\hline \multirow[b]{2}{*}{ Fat subtypes } & \multicolumn{2}{|c|}{ Entire population ( $n 35372$ ) } & \multicolumn{2}{|c|}{ Pre-menopausal ( $n 15$ 951) } & \multicolumn{2}{|c|}{ Post-menopausal ( $n$ 17 781) } \\
\hline & $\mathrm{HR}(\mathrm{CI})$ & $P$-value & $\mathrm{HR}(\mathrm{CI})$ & $P$-value & $\mathrm{HR}(\mathrm{CI})$ & $P$-value \\
\hline Total fat & $1.26(0.83-1.90)$ & 0.712 & $1.85(0.99-3.48)$ & 0.235 & $0.99(0.57-1.70)$ & 0.902 \\
\hline SFA & $1.20(0.83-1.73)$ & 0.751 & $1.26(0.72-2.18)$ & 0.602 & $1.17(0.72-1.91)$ & 0.912 \\
\hline MUFA & $0.85(0.57-1.25)$ & 0.910 & $1.16(0.65-2.07)$ & 0.675 & $0.67(0.40-1.14)$ & 0.599 \\
\hline PUFA & $1.18(0.80-1.75)$ & 0.639 & $1.62(0.89-2.93)$ & 0.465 & $0.96(0.57-1.62)$ & 0.945 \\
\hline
\end{tabular}

HR = hazard ratio

In this study, we found no significant association between intakes of total fat, SFA, MUFA and PUFA with breast cancer in the entire cohort, or in pre- and post-menopausal women or women with and without family history of breast cancer.

1. National Breast Cancer Foundation Inc (2007). What is breast cancer? http://www.nationalbreastcancer.org/AboutBreast-Cancer/What-Is-Breast-Cancer/.

2. Cancer Research UK (2007) UK breast cancer incidence. http://info.cancerresearchuk.org/cancerstats/types/breast/incidence/.

3. Prentice RL \& Sheppard L (1990) Cancer Causes Control 1, 81-97.

4. Fay MP, Freedman LS, Clifford CK et al. (1997) Cancer Res 57, 3979-3988.

5. Harrison RA \& Waterbor JW (1999) Cancer Detect Prev 23, 97-106.

6. Knekt P, Albanes D, Seppanen R et al. (1990) Am J Clin Nutr 52, 903-908.

7. Lof M, Sandin S, Lagiou P et al. (2007) Br J Cancer 97, 1570-1576. 